\title{
Infective endocarditis in patients with diabetes: More than meets the eye?
}

\author{
David D. Yuh, MD, FACS, FACC
}

From the Department of Surgery, Stamford Hospital, Stamford, Conn.

Disclosures: Author has nothing to disclose with regard to commercial support.

Received for publication Sept 17, 2017; accepted for publication Sept 27, 2017; available ahead of print Oct 26, 2017.

Address for reprints: David D. Yuh, MD, FACS, FACC, Department of Surgery, Stamford Hospital, One Hospital

Plaza, PO Box 9317, Stamford, CT 06904 (E-mail: DYuh@stamhealth.org).

J Thorac Cardiovasc Surg 2018;155:1030-1

$0022-5223 / \$ 36.00$

Copyright $@ 2017$ by The American Association for Thoracic Surgery

https://doi.org/10.1016/j.jtcvs.2017.09.121

In their multicenter retrospective study reported in this issue of the Journal, Yoshioka and colleagues ${ }^{1}$ seek to differentiate clinical outcomes between patients with and without diabetes undergoing surgical therapy for infective endocarditis (IE). The study differentiates itself from others in that it focuses solely on patients with IE treated with surgery and examines midterm to long-term clinical outcomes. Furthermore, the patient cohort studied is contemporary, which lends itself to observations and conclusions resulting from current "real-world" treatment strategies, including perioperative antibiotic regimens. The overarching conclusion of this study confirms the intuitive notion that patients with diabetes fare worse than those without when undergoing surgery for IE. When one delves deeper into the substance of the results, however, several important corollary conclusions of this study carry the potential to affect the management of IE in patients with diabetes.

First, Yoshioka and colleagues ${ }^{1}$ effectively identify diabetes mellitus as an independent risk factor for postoperative infection-related mortality among patients with IE. By means of multivariate and propensity score analyses, Yoshioka and colleagues ${ }^{1}$ address the argument that their observations might have stemmed from one or more covariates commonly observed among patients with diabetes, including higher body mass index, long-term hemodialysis, hyperglycemia, and more extensive atherosclerotic coronary disease. This finding may provide practical prognostic implications and modify preoperative risk stratification of patients with diabetes.

Second, the study revealed that there was a higher prevalence of Staphylococcus species and a higher proportion of patients requiring annular or aortic root reconstruction among patients with diabetes and IE, with longer operative and aortic crossclamp times, more platelet transfusions, and a greater need for extracorporeal membrane oxygenation and continuous venovenous hemodialysis support. These findings prompt the question of whether patients with diabetes who are seen for IE should be treated more aggressively than those without diabetes, including earlier operative intervention, because of the higher likelihood of

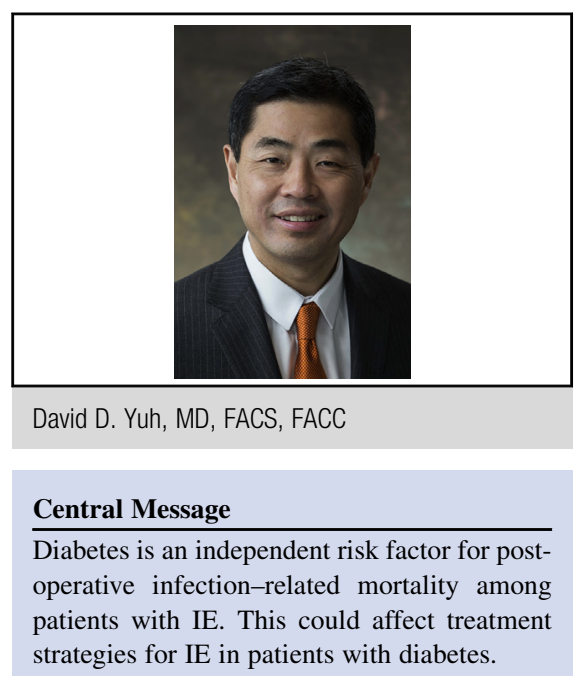

See Article page 1021.

more extensive structural damage from infections with more virulent Staphylococcus infections than from those arising from Streptococcus or other bacteria. Although the current American Association for Thoracic Surgery consensus guidelines for the surgical treatment of IE, cogently documented by Coselli and colleagues, ${ }^{2}$ clearly acknowledge the virulence of Staphylococcus species, future iterations of these guidelines armed with additional evidence may define special treatment considerations for patients with diabetes, given that such infections may be even more virulent in these immunocompromised patients.

Finally, a higher incidence of "recurrent" IE in patients with diabetes was noted in this study. Strictly speaking, recurrent IE implies a resurgence of the index pathogen after treatment. Yoshioka and colleagues ${ }^{1}$ used a broader definition to include a minority ( 5 of 21) subset of patients who incurred endocarditis with a different bacterial species. Regardless, this raises the longstanding controversial issue pertaining to the duration and composition of postoperative antibiotic treatment in patients with IE. Is more sustained, perhaps even lifelong, prophylactic antibiotic therapy warranted in patients with diabetes and IE? Do the immunologic or other features of diabetes place these patients in a different treatment category? Unfortunately, the regimens of postoperative antibiotics varied considerably in this study, which Yoshioka and colleagues ${ }^{1}$ rightly identified as a study limitation. Despite the inherent limitations of this retrospective analysis, however, Yoshioka and 
colleagues ${ }^{1}$ raise issues that may potentially affect treatment strategies for IE in patients with diabetes.

\section{References}

1. Yoshioka D, Toda K, Yokoyama J-Y, Matsuura R, Miyagawa S, Kainuma S, et al. Diabetes mellitus adversely affects mortality and recurrence after valve surgery for infective endocarditis. J Thorac Cardiovasc Surg. 2018;155: 1021-9.e5.

2. Coselli JS, Petersson GB, Hussain ST, Griffin B, Blackstone EH, Gordon SM, et al; AATS Surgical Treatment of Infective Endocarditis Consensus Guidelines Writing Committee. 2016 The American Association for Thoracic Surgery (AATS) consensus guidelines: surgical treatment of infective endocarditis: executive summary. J Thorac Cardiovasc Surg. 2017;153:1241-58.e29. 